\title{
Demonstration of (In, Ga)N/GaN Core-Shell Micro Light-Emitting Diodes Grown by Molecular Beam Epitaxy on Ordered MOVPE GaN Pillars
}

Steven Albert, Ana Bengoechea-Encabo, Johannes Ledig, Tilman Schimpke, Miguel A. Sánchez-Garcia, Martin Strassburg, Andreas Waag, and Enrique Calleja

ABSTRACT: This work reports on the growth of (In, Ga)N core-shell micro pillars by plasma-assisted molecular beam epitaxy using an ordered array of GaN cores grown by metal organic vapor phase epitaxy as a template. Upon (In, Ga)N growth, core-shell structures with emission at around $3.0 \mathrm{eV}$ are formed. With spatially resolved cathodoluminescence, an increasing In content toward the pillar top is found to be present in the (In, Ga)N shell, as indicated by a shift of CL peak position from $3.2 \mathrm{eV}$ at the shell bottom to $3.0 \mathrm{eV}$ at the
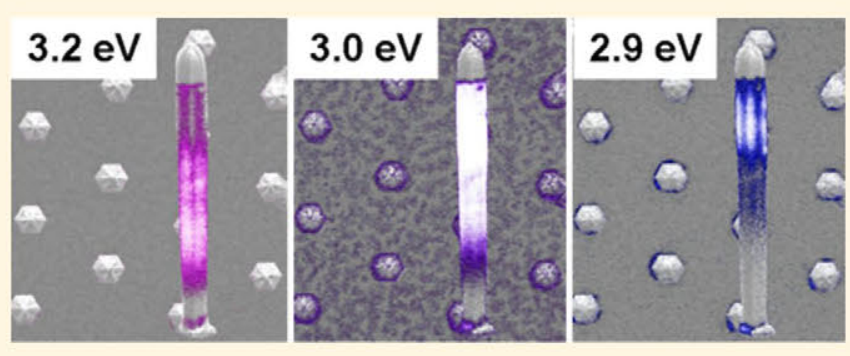
shell top. Further, the fabrication of a core-shell pin structure is demonstrated. Spatially resolved electroluminescence measurements performed on individual pillars confirm electroluminescence from the (In, Ga) N shell (lateral diode) at around $3.0 \mathrm{eV}$, as well as from the pillar top facet (axial diode) at around $2.3 \mathrm{eV}$.

\section{INTRODUCTION}

(In, Ga) N alloys are exceptional candidates for optoelectronic devices, such as solar cells and light-emitting diodes (LED), because of their wide, direct band gap ranging from 0.7 to 3.4 $\mathrm{eV} .^{1-3}$ Most of the research effort on these materials focused so far on planar (In, Ga) N/GaN quantum well structures grown along the $c$-direction, which generally suffer from a high density of nonradiative defects for higher In contents because of the lattice mismatch between $\mathrm{GaN}$ and ( $\mathrm{In}, \mathrm{Ga}$ ) N and the lack of affordable native substrates. These limitations can be overcome with the use of nanocolumnar structures whose large surface to volume ratio allows for efficient strain relaxation without generation of extended defects. The self-assembled (SA) and selective area growth (SAG) of GaN nanocolumns (NCs) have been demonstrated by various epitaxial techniques, such as metal organic vapor phase epitaxy (MOVPE), plasma-assisted molecular beam epitaxy (PAMBE), and hydride vapor phase epitaxy (HVPE). ${ }^{3-6}$ In addition, the growth of SA and SAG axial (In, Ga)N/GaN NCs by PAMBE has been demonstrated. $^{7-12}$

To exploit an enhanced emission/absorption and a reduced (or zero) internal electric field in NC-based LEDs, the growth of high-aspect ratio (In, Ga)N/GaN core-shell structures was addressed and achieved by MOVPE. ${ }^{13-19}$ Aside from a large lateral surface, the distance between NCs (pitch) needed to accommodate micrometer-size phosphors for light conversion (white LEDs) leads to NC diameters and pitch values in the micrometer range. Hereafter, these structures will be termed micro pillars (MP).

The development of (In, Ga)N/GaN core-shell growth by PAMBE would be relevant because of its potential for the growth of higher-In content shell layers that are not straightforwardly obtained by MOVPE. However, because of rather small growth rates in PAMBE, the SAG of MPs with a high aspect ratio (several micrometers high) is not practical. A different approach based on the PAMBE growth of GaN and (In, Ga) $\mathrm{N}$ shell layers over ordered $\mathrm{GaN}$ cores etched from a planar GaN template was recently demonstrated. ${ }^{20}$ This approach has some drawbacks like the limitation in terms of core heights that depend on the ability to deep etch cylindrically shaped GaN cores and the fact that their quality is the same as that of the template (i.e., dislocations, yellow band, etc.).

This work demonstrates the growth of (In, Ga)N/GaN MP core-shell micro-LED structures by PAMBE using high-aspect ratio (>10) GaN pillars, grown by MOVPE, as templates. 


\section{EXPERIMENTAL SECTION}

All samples were grown in a Riber Compact 21 PAMBE system equipped with an rf-plasma source for active nitrogen and standard Knudsen cells for $\mathrm{Ga}, \mathrm{In}, \mathrm{Si}$, and $\mathrm{Mg}$. All effusion cells and the plasma source are mounted with an angle of $\sim 45^{\circ}$ with respect to the substrate top surface. The separation among the cells is $60^{\circ}$. The sample rotation during growth is around $14 \mathrm{rpm}$. The molecular fluxes were calibrated in (0001) GaN growth rate units (nanometers per minute) for $\mathrm{Ga}$ and nitrogen, and (0001) InN growth rate units for In. The shell overgrowth was performed on SAG MOVPE GaN MPs arrays grown on n-type $\mathrm{GaN} /$ sapphire masked with patterned $\mathrm{SiO}_{x}$ (OSRAM Opto Semiconductors) having a diameter of $550 \mathrm{~nm}$, a length of $7.3 \mu \mathrm{m}$, and a pitch of $2.4 \mu \mathrm{m}$ with an aspect ratio of $13 .{ }^{21}$ Two series of samples, A and B, were grown, and details are listed in Table 1. Before growth, the MOVPE pillars were cleaned ex situ with pyrrolidone and inside the growth chamber by a two-step thermal cycle for $30 \mathrm{~min}$ at 350 and $550{ }^{\circ} \mathrm{C}$.

\section{Table 1. Growth Conditions for Samples of Series A and B}

\begin{tabular}{clll} 
sample & \multicolumn{1}{c}{ grown layers } & \multicolumn{1}{c}{ substrate } & \multicolumn{1}{c}{$T_{\text {sample }}\left({ }^{\circ} \mathrm{C}\right)$} \\
A1 & GaN $(1 \mathrm{~h})+\operatorname{InGaN}(2 \mathrm{~h})$ & MOVPE SAG & $860 / 625$ \\
& & GaN & \\
A2 & $+\operatorname{InGaN}(2 \mathrm{~h})$ & $\mathrm{A} 1$ & 625 \\
$\mathrm{~A} 3$ & $+\operatorname{InGaN}(2 \mathrm{~h})$ & $\mathrm{A} 2$ & 600 \\
$\mathrm{~B} 1$ & $\mathrm{GaN}: \mathrm{Si}(1 \mathrm{~h})+\operatorname{InGaN}(2 \mathrm{~h})+$ & MOVPE SAG & $860 / 625 / 625$ \\
& $\mathrm{GaN}: \mathrm{Mg}(1 \mathrm{~h})$ & $\mathrm{GaN}$ & \\
\hline
\end{tabular}

The purpose of series A was to demonstrate (In, Ga)N core-shell growth by PAMBE, as well as to check the incorporation of In into the (In, Ga)N shell. In sample A1, a GaN shell was first grown for $1 \mathrm{~h}$ with a Ga flux $\left(\Phi_{\mathrm{Ga}}\right)$ of $18 \mathrm{~nm} / \mathrm{min}\left(1.3 \times 10^{15}\right.$ atoms s $\left.\mathrm{sm}^{-1}\right)$ and a $\mathrm{N}$ flux $\left(\Phi_{\mathrm{N}}\right)$ of $5 \mathrm{~nm} / \mathrm{min}\left(3.7 \times 10^{14}\right.$ atoms $\left.\mathrm{s}^{-1} \mathrm{~cm}^{-2}\right)$ at a growth temperature of $860^{\circ} \mathrm{C}$ (measured with a thermocouple) to provide a clean GaN surface before growing (In, Ga)N for $2 \mathrm{~h}$ (sample A1). The $(\mathrm{In}, \mathrm{Ga}) \mathrm{N}$ shell was grown with a $\Phi_{\mathrm{Ga}}$ of $3 \mathrm{~nm} / \mathrm{min}\left(2.2 \times 10^{14}\right.$ atoms $\left.\mathrm{s}^{-1} \mathrm{~cm}^{-2}\right)$, a $\Phi_{\mathrm{In}}$ of $9.6 \mathrm{~nm} / \mathrm{min}\left(5.1 \times 10^{14}\right.$ atoms s $\left.\mathrm{cm}^{-1}\right)$, and a $\Phi_{\mathrm{N}}$ of $12 \mathrm{~nm} / \mathrm{min}\left(8.8 \times 10^{14}\right.$ atoms $\left.\mathrm{s}^{-1} \mathrm{~cm}^{-2}\right)$ at $625{ }^{\circ} \mathrm{C}$. After characterization, sample A1 was reloaded into the MBE chamber, and further (In, Ga) N growth proceeded for an additional $2 \mathrm{~h}$ under the same conditions (sample A2). Once characterized, this procedure was repeated once more (sample $\mathrm{A} 3$ ) using the same fluxes but a lower growth temperature of $600^{\circ} \mathrm{C}$. Before being reloaded, the sample was cleaned each time ex situ using pyrrolidone at $75{ }^{\circ} \mathrm{C}$ and then inside the growth chamber by a two-step thermal cycle for $30 \mathrm{~min}$ at 350 and $400{ }^{\circ} \mathrm{C}$. During the thermal cleaning at $400{ }^{\circ} \mathrm{C}$, a nitrogen flux of 5 $\mathrm{nm} / \mathrm{min}$ was provided.

After growth, the samples were characterized by scanning electron microscopy (SEM) using a FEI Inspect F50 instrument additionally equipped with a Gatan Mono CL4 unit allowing for cathodoluminescence $(\mathrm{CL})$ measurements and photoluminescence (PL) spectroscopy using a $\mathrm{He}-\mathrm{Cd}$ laser $(325 \mathrm{~nm})$ with a power density of $\sim 1 \mathrm{~W} /$ $\mathrm{cm}^{2}$.

Series B consisted of a single (p-i-n) structure (B1) with GaN:Mg/ (In, Ga)N/GaN:Si stacked sections to demonstrate the feasibility of core-shell micro-LEDs grown by PAMBE. The GaN and (In, Ga)N sections were grown in the same way as in sample Al except for the use of Si doping during the $\mathrm{GaN}$ growth ( $\mathrm{Si}$ cell temperature of 950 $\left.{ }^{\circ} \mathrm{C}\right)$. Then, a GaN: $\mathrm{Mg}$ shell layer was grown for $1 \mathrm{~h}$ at $625^{\circ} \mathrm{C}\left(\Phi_{\mathrm{Ga}}=\right.$ $8 \mathrm{~nm} / \mathrm{min}$, and $\Phi_{\mathrm{N}}=12 \mathrm{~nm} / \mathrm{min}$ ) with a $\mathrm{Mg}$ cell temperature of 365 ${ }^{\circ} \mathrm{C}$. At this point, it must be noted that the $\mathrm{GaN}: \mathrm{Mg}$ was grown at a sample temperature much lower than that of the $\mathrm{GaN}: \mathrm{Si}$ to prevent and/or reduce $\mathrm{InN}$ decomposition.

EL of the MP-LEDs was measured using a piezo-driven tungsten probe tip mounted inside a CL-SEM instrument. The second contact on top of the $\mathrm{n}-\mathrm{GaN}$ buffer was made with the SEM sample holder by clamping the sample with a spring wire. To enhance the conductivity, a small drop of $\mathrm{Ag}$ lacquer was used at the contact point. The contact position of the tungsten probe tip was depicted by means of secondary electrons (SE), while the e-beam was blocked during the EL measurements. With this technique, spatially resolved excitation can be performed; hence, local properties with respect to the contact position could be extracted.

\section{RESULTS AND DISCUSSION}

Figure 1 shows top and cross-section SEM pictures of the SAG MOVPE MPs and sample A1. Figure $1 \mathrm{~b}$ shows a hexagonal
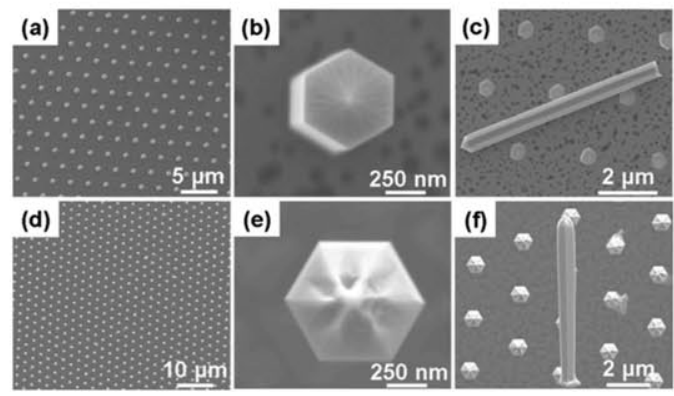

Figure 1. SEM pictures of $(a-c)$ MOVPE-grown GaN pillars and (df) sample A1. A diameter increase toward the MP top is observed upon $(\mathrm{In}, \mathrm{Ga}) \mathrm{N} / \mathrm{GaN}$ overgrowth (f).

shape with smooth sidewalls of the MOVPE GaN MPs. The subsequent growth of (In, Ga)N/GaN leads to a clear increase in diameter from the initial MOVPE GaN MPs $(550 \mathrm{~nm})$ to $640 \mathrm{~nm}$ at the bottom of the MPs and $770 \mathrm{~nm}$ at the top of the MPs in sample A1.

The low-temperature (LT) PL spectrum of sample A1 (Figure 2a) reveals a main emission peak at around $3.1 \mathrm{eV}$ corresponding to an In content of $\sim 10 \% .^{22}$ In addition, a

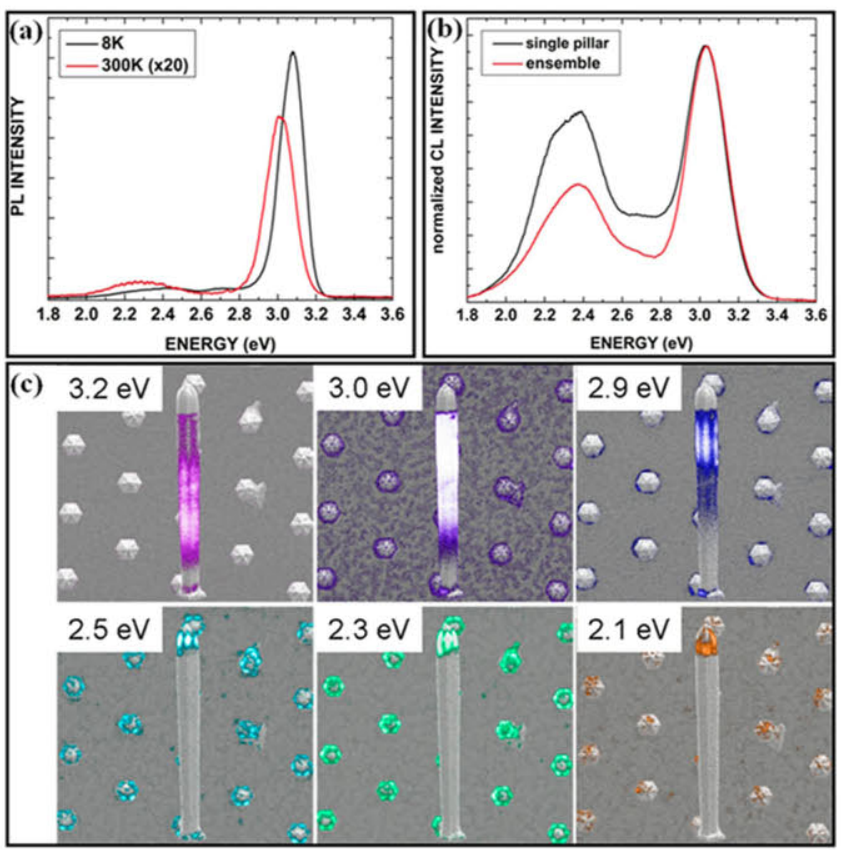

Figure 2. (a) Room-temperature (RT) and $8 \mathrm{~K}$ PL spectra of sample A1 measured under an excitation angle of $40^{\circ}$. (b) RT CL spectra of a single MP and an ensemble of 300 MPs of sample A1 measured in top view. (c) Spatially resolved CL measurements of sample Al measured in cross section showing the overlaid monochromatic CL intensity images (color) with the secondary electron image (grayscale) of an individual MP. 
second (In, Ga)N-related broader emission centered at $\sim 2.3 \mathrm{eV}$ can be observed. The internal quantum efficiency (IQE) of the emission centered at $3.1 \mathrm{eV}$, estimated from the low- to roomtemperature PL intensity ratio, is $\sim 4 \%$, which is in the range of the PL-IQE of axial SAG InGaN/GaN NCs grown on GaN/ sapphire templates under similar conditions. ${ }^{10}$ Weak localization is expected in a bulklike InGaN layer (no quantum confinement) with $10 \% \mathrm{In}$, so that recombination via nonradiative channels is pronounced, adding to a low IQE value. The PL-IQE of the broader emission in the range between 2.1 and $2.8 \mathrm{eV}$ is estimated to be $\sim 6 \%$. It is shown in ref 20 that the PL-IQE of GaN overgrown GaN pillars is around 4-5\%; thus, the pumping of InGaN by emission from the $\mathrm{GaN}$ core is thought to have no major impact on the final PL-IQEs.

Figure $2 \mathrm{~b}$ shows RT CL spectra of a single MP and an ensemble of $\sim 300 \mathrm{MPs}$ from sample A1. The first apparent fact is that both spectra are quite similar, pointing to a high uniformity of MPs. In addition, two emission peaks contribute to the spectra at 2.3 and $3.0 \mathrm{eV}$, in agreement with those observed by PL. Spatially resolved SEM-CL measurements (Figure 2c) reveal that the emission at $3.0 \mathrm{eV}$ originates at the MP side facets (m-plane), whereas the emission at $2.3 \mathrm{eV}$ comes from the topmost region of the MPs. These two (In, $\mathrm{Ga}) \mathrm{N}$ emission peaks are attributed to a different In incorporation depending on the crystal plane, i.e., m-plane for the lateral shell and $\{10-11\}$ planes for the top region. This agrees with findings of Wernicke et al., who reported the level of incorporation of In on the $\{10-11\}$ planes to be significantly higher than on the m-plane in the case of MOVPE growth. ${ }^{23}$ Furthermore, it has to be noted that similar results were found for (In, Ga)N/GaN core-shell structures grown on etched $\mathrm{GaN}$ MPs where the GaN pillar top was formed by c- and $\mathrm{r}$ planes. ${ }^{20}$ In addition, a red-shift of the (In, Ga)N shell-related emission toward the MP top can be observed (Figure 2c, upper side), indicating the presence of an In composition gradient.

In composition gradients are generally attributed to thermal InN decomposition and In segregation toward the NC top side as reported for axial SAG (In, Ga)N/GaN NCs. ${ }^{10}$ However, the composition gradient observed in Figure $2 c$ could also be due to variations in the local III/V ratio at the side facet along the MP or lattice pulling effects due to strain generated when growing InGaN. Lattice pulling was found to lead to a lower level of incorporation of In in regions with high strain. ${ }^{24}$ Kong et al. reported a pulling region width of around $50 \mathrm{~nm}$ for an In content of $25.5 \%$ in axial SAG (In, Ga)N/GaN NCs. ${ }^{25}$ The presence of lattice pulling effects is suggested by the shell thickness increase toward the MP top (bottom diameter of 640 $\mathrm{nm}$ to top one of $770 \mathrm{~nm}$ ) as observed in Figure 1f. RT CL point spectra along a single MP sidewall (Figure 3) clearly show the increase in In composition toward the top side. At the MP bottom (point P1), the CL spectrum is dominated by the $\mathrm{GaN}$ emission while emission from the (In, Ga)N shell is barely seen, meaning that this shell, if present, must be very thin.

To further check for the presence of lattice pulling effects, sample A2, with a double (In, Ga)N growth time (an additional $2 \mathrm{~h}$ ) compared to that of sample Al, was grown. SEM pictures in Figure 4 show the evolution of the diameter of individual MPs, where the top diameter changes from $770 \mathrm{~nm}$ (sample A1 grown for $2 \mathrm{~h}$ ) to $970 \mathrm{~nm}$ (sample $\mathrm{A} 2$ grown for $4 \mathrm{~h}$ ) as a consequence of the increased growth time.

If pulling effects were present, a thicker (In, Ga)N shell would lead to an increasing average In content, as well as to a

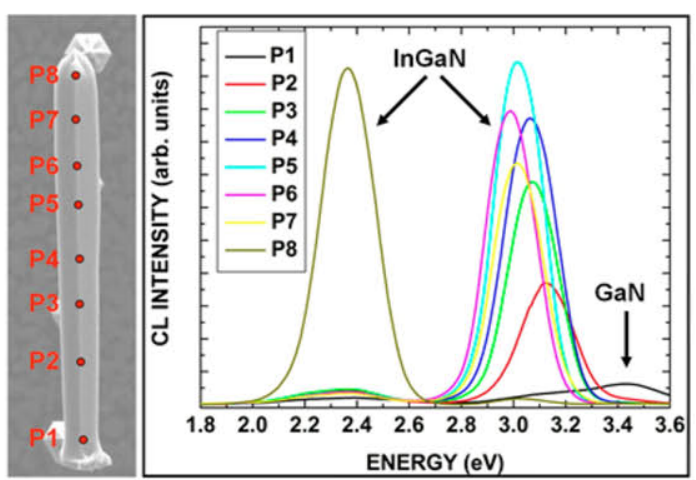

Figure 3. RT CL point measurements of a single MP from sample A1, showing an emission energy red-shift toward the MP top along its sidewall, as a result of an increasing level of In incorporation.
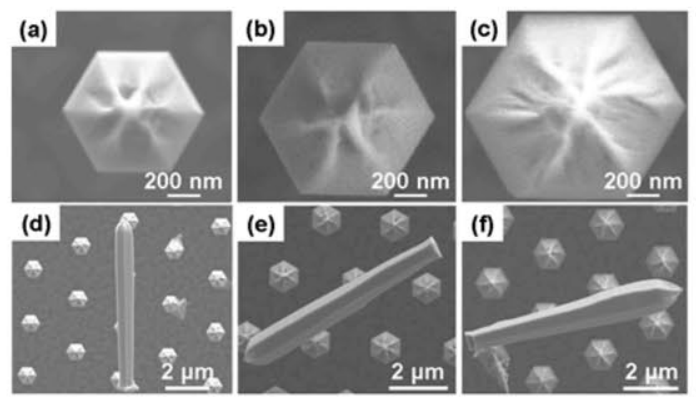

Figure 4. Top view SEM pictures of a single MP of samples (a) A1, (b) A2, and (c) A3. Cross-sectional SEM pictures of samples (d) A1, (e) A2, and (f) A3.

reduction in the emission energy red-shift toward the MP top (P1 to P7). RT CL point measurements along single MPs of samples A1 and A2 shown in Figure 5a reveal that, with an increase in thickness, the same In gradient and average In

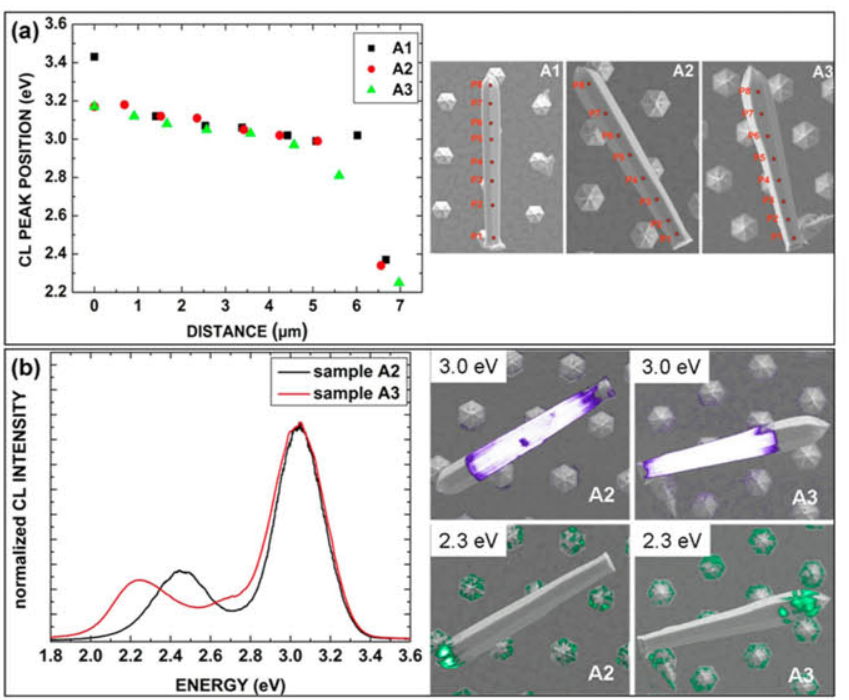

Figure 5. (a) RT CL point measurements of samples $\mathrm{A} 1-\mathrm{A} 3$ and cross-section SEM pictures of measured MPs with an indication of the point at which measurements were performed. (b) RT CL measurements of samples $\mathrm{A} 2$ and $\mathrm{A} 3$ and spatially resolved $\mathrm{CL}$ measurements of samples A2 and A3 showing the overlaid monochromatic CL intensity images with secondary electron contrast of an individual MP. 
content are present in sample $\mathrm{A} 2$, as indicated by the negligible change in peak positions. On the basis of this result, lattice pulling effects can be ruled out as the origin of the increasing In content toward the MP top in the (In, Ga)N shell.

To study the potential effects of InN thermal decomposition and In desorption on the shell In-graded composition and average In content, another ( $\mathrm{In}, \mathrm{Ga}) \mathrm{N}$ shell was grown at a lower temperature $\left(600^{\circ} \mathrm{C}\right)$ in sample A3. The SEM images in Figure 4 show a diameter increase from $970 \mathrm{~nm}$ (sample A2) to $1.34 \mu \mathrm{m}$ (sample A3) measured at the MP top. Aside from this diameter increase, a hexagonal shape with well-defined $\mathrm{m}$-plane sidewalls is observed. Given the nominally nitrogen rich conditions during the (In, Ga) $\mathrm{N}$ growth (III/V $\approx 0.83$ ), a decrease in growth temperature should in principle lead to an increasing average In content (due to reduced $\mathrm{InN}$ decomposition and In desorption) and a concomitant reduction of the In gradient in the shell toward the MP top.

The RT CL spectrum of sample A3 (Figure $5 b$ ) shows a main emission peak at $3.0 \mathrm{eV}$, identical to that of sample A2, indicating that a reduction of growth temperature does not have any impact on the average In content of the (In, Ga)N shell. Only the CL emission from the MP top (growth on cplane), at around $2.2 \mathrm{eV}$, reveals an increase in the In content for sample A3. This finding most likely relates to the accumulation of In close to and at the MP top.

Spatially resolved CL measurements (Figure $5 \mathrm{~b}$ ) confirm that the emission at $3.0 \mathrm{eV}$ originates from the MP sidewalls while the low-energy emission at $2.2 \mathrm{eV}$ comes from the MP top. Furthermore, RT CL point measurements in sample A3 (Figure 5a) indicate again the presence of an In gradient toward the MP top, which is similar to that found in samples Al and A2. The independence of incorporation of In into the sidewall shell from the growth temperature is surprising given the growth temperatures of 625 and $600{ }^{\circ} \mathrm{C}$, which are well above the onset temperature of $\mathrm{InN}$ decomposition for both $\mathrm{N}$-face (around $520^{\circ} \mathrm{C}$ ) and In-face (around $410^{\circ} \mathrm{C}$ ) InN as well as In desorption. ${ }^{26,27}$ At this point, the origin of this behavior is unclear, and further studies are needed to resolve this issue. However, on the basis of these findings, it can be concluded that neither lattice pulling effects, decomposition, nor In desorption is responsible for the average In content or In gradient observed in the shell; hence, additional effects have to be considered. Depending on the local III/V ratio at the growth front (m-plane), the In content is in principle determined by either the supplied amount of nitrogen under N-limited conditions or the provided In flux under metal-limited conditions. $^{28}$ In the case of InGaN core-shell structures, the local III/V ratio at the growth front may additionally be affected by a variety of other factors. The first is the geometrical arrangement of the growth plane with respect to the impinging fluxes (effusion cells and plasma source) in combination with the sample rotation. All effusion cells and the plasma source are mounted with an angle of $45^{\circ}$ with respect to the substrate top surface. Because of that, every molecular beam impinges at different MP facets as a function of time, leading to a growth process similar to migration-enhanced epitaxy (MME). ${ }^{29}$ In addition, diffusion anisotropy of the metal adatoms on the $\mathrm{m}$ plane sidewalls as well as the shadowing effect by surrounding MPs has to be considered. ${ }^{30,31}$ These effects would also account for the observed tapering of the MPs, which can be understood as a result of different lateral growth rates of the ( $\mathrm{In}, \mathrm{Ga}) \mathrm{N}$ shell along the pillar. The hexagonal shape of the MPs is thought to be maintained due to surface energy minimization that favors the hexagonal shape with m-plane sidewalls. Here it is worth noting that upon InGaN shell overgrowth of a circular GaN pillar under similar conditions a transition from a circular shape to a hexagonal shape has been observed. ${ }^{20}$

The potential of PAMBE-grown core-shell MPs for LED applications was checked by growing a p-i-n micro LED (sample B1), as shown by the SEM top and side view in panels $a$ and $b$ of Figure 6. Starting from the same structure as that in
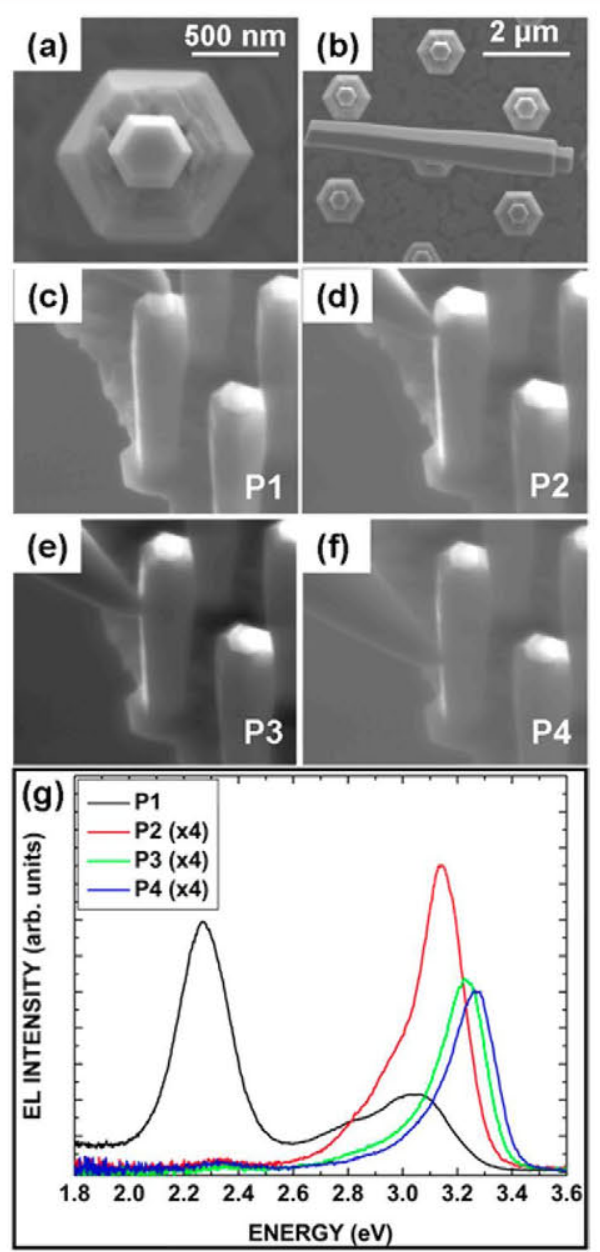

Figure 6. (a) Top view and (b) cross-section SEM images of a single core-shell micro LED (sample B1). (c-f) SEM images at $30^{\circ}$ tilt showing the probe tip contact at four different positions on the top and sidewall of a single micro LED to obtain EL. (g) EL spectra taken at respective positions $\mathrm{P} 1-\mathrm{P} 4$ with an injection current of $50 \mu \mathrm{A}$.

sample A1, the micro LEDs include a Mg-doped top GaN shell layer. The micro LED morphology shows a flat top with an outer and inner hexagonal structure, in contrast to the pyramidal top in sample A1, which is assumed to relate to the growth of the Mg-doped shell.

EL measurements were performed by current injection via a probe tip placed at the sidewall and top facet of the MP-LED, as shown in panels $\mathrm{c}-\mathrm{f}$ of Figure 6 . Because of the absence of a current spreading layer, the current injection takes place at an area around the contact point that results in a localized injection of carriers into the active region.

EL spectra obtained with a driving current of $50 \mu \mathrm{A}$ measured at different contact points on the micro LED are shown in Figure 6g. One can see that while the spectrum taken at P1 (pillar top) shows a main emission peak at $\sim 2.3 \mathrm{eV}$ 
together with a second emission peak at $\sim 3.0 \mathrm{eV}$, spectra taken at points $\mathrm{P} 2-\mathrm{P} 4$ show emission at $\sim 3.0 \mathrm{eV}$ with a decreasing emission energy toward the pillar top. On the basis of the results for samples of series $A$, the higher-energy peak observed at P1 is believed to originate at the lateral shell (m-plane) close to the MP top, which is excited by injected carriers from the top contact, while the emission at $2.3 \mathrm{eV}$ relates to an axial diode grown on the pillar top. The observed red-shift of the EL emission energy (from P4 to P1) coming from the (In, Ga)N shell (lateral diode) is in agreement with the findings presented for samples of series A, where an In gradient toward the MP top was found to be present in the (In, Ga) N shell (Figure 5a).

In addition, it must be noted that the EL emission peak of the lateral diode shows a pronounced low-energy tail, which is absent in the EL emission peak of the axially grown diode. On the basis of results presented in ref 32 , this difference in peak shape can be attributed to the presence of different distributions, densities, and depths of localized states in both regions depending on the In content.

\section{CONCLUSION}

In summary, (In, Ga) N overgrowth was performed by PA-MBE over ordered arrays of MOVPE-grown GaN pillars, leading to (In, Ga)N core-shell structures with aspect ratios of around 13. In spatially resolved CL, a red-shift of the CL emission of the (In, Ga) N shell toward the top was observed, indicating an increasing In content in the shell toward the top. Furthermore, the fabrication of core-shell pin structures using PAMBE was demonstrated. Spatially resolved EL measurements, performed on an individual pillar, confirmed emission from the (In, Ga)N shell at around $3.0 \mathrm{eV}$. In addition, a second pin junction was formed at the pillar top, emitting at around $2.3 \mathrm{eV}$.

\section{AUTHOR INFORMATION}

\section{Corresponding Author}

*E-mail: salbert@isom.upm.es.

\section{Author Contributions}

S.A and A.B.-E. contributed equally to this work.

\section{Notes}

The authors declare no competing financial interest.

\section{ACKNOWLEDGMENTS}

We acknowledge partial financial support by EU FP7 Contract GECCO 280694-2 and by Spanish Projects CAM/P2009/ESP1503 and MICINN MAT2011-26703.

\section{REFERENCES}

(1) Nakamura, S.; Fasol, G.; Pearton, S. J. The Blue Laser Diode: The Complete Story; Springer: Berlin, 2000.

(2) Yam, F. K; Hassan, Z. Superlattices Microstruct. 2008, 43, 1-23.

(3) Kuykendall, T.; Ulrich, P.; Aloni, S.; Yang, P. Nat. Mater. 2007, 6, 951-956.

(4) Calleja, E.; Sánchez-García, M. A.; Sánchez, F. J.; Calle, F.; Naranjo, F. B.; Muñoz, E.; Jahn, U.; Ploog, K. Phys. Rev. B 2000, 62, 16826-16834.

(5) Kishino, K.; Sekiguchi, H.; Kikuchi, A. J. Cryst. Growth 2009, 311, 2063-2068.

(6) Koester, R; Hwang, J. S.; Durand, C.; Le Si Dang, D.; Eymery, J. Nanotechnology 2010, 21, 1-9.

(7) Guo, W.; Zhang, M.; Banerjee, A.; Bhattacharya, P. Nano Lett. 2010, 10, 3355-3359.

(8) Sekiguchi, H.; Kishino, K.; Kikuchi, A. Appl. Phys. Lett. 2010, 96, 231104.
(9) Albert, S.; Bengoechea-Encabo, A.; Lefebvre, P.; Sánchez-García, M. A.; Calleja, E.; Jahn, U.; Trampert, A. Appl. Phys. Lett. 2011, 99, 131108 .

(10) Albert, S.; Bengoechea-Encabo, A.; Sanchez-Garcia, M. A.; Calleja, E.; Jahn, U. J. Appl. Phys. 2013, 113, 114306.

(11) Albert, S.; Bengoechea-Encabo, A.; Kong, X.; Sanchez-Garcia, M. A.; Calleja, E.; Trampert, A. Appl. Phys. Lett. 2013, 102, 181103.

(12) Albert, S.; Bengoechea-Encabo, A.; Sanchez-Garcia, M. A.; Kong, X.; Trampert, A.; Calleja, E. Nanotechnology 2013, 24, 1-8.

(13) Qian, F.; Li, Y.; Gradecak, S.; Wang, D.; Barrelet, C. J.; Lieber, C. M. Nano Lett. 2004, 4, 1975-1979.

(14) Qian, F.; Gradecak, S.; Li, Y.; Wen, C. Y.; Lieber, C. M. Nano Lett. 2005, 5, 2287-2291.

(15) Dong, Y.; Tian, B.; Kempa, T. J.; Lieber, C. M. Nano Lett. 2009, 9, 2183-2187.

(16) Bavencove, A. L.; Salomon, D.; Lafossas, M.; Martin, B.; Dussaigne, A.; Levy, F.; Andre, B.; Ferret, F.; Durand, C.; Eymery, J.; Dang, L. S.; Gilet, P. Electron. Lett. 2011, 47, 765-767.

(17) Koester, R.; Hwang, J. S.; Salomon, D.; Chen, X.; Bougerol, C.; Barnes, J. P.; Le Si Dang, D.; Rigutti, L.; de Luna Bugallo, A.; Jacopin, G.; Tchernycheva, M.; Durand, C.; Eymery, J. Nano Lett. 2011, 11, $4839-4845$.

(18) Jacopin, G.; de Luna Bugallo, A.; Lavenus, P.; Rigutti, L.; Julien, F. H.; Zagonel, L. F.; Kociak, M.; Durand, C.; Salomon, D.; Jun Chen, X.; Eymery, J.; Tchernycheva, M. Appl. Phys. Express 2012, 5, 014101.

(19) Wierer, J. J.; Li, Q.; Koleske, D. D.; Lee, S. R.; Wang, G. T. Nanotechnology 2012, 23, 1-6.

(20) Albert, S.; Bengoechea-Encabo, A.; Sabido-Siller, M.; Müller, M.; Schmidt, G.; Metzner, S.; Veit, P.; Bertram, F.; Sanchez-Garcia, M. A.; Christen, J.; Calleja, E. J. Cryst. Growth 2014, 392, 5-10.

(21) Mandl, M.; Wang, X.; Schimpke, T.; Kölper, Ch.; Binder, M.; Ledig, J.; Waag, A.; Kong, X.; Trampert, A.; Bertram, F.; Christen, J.; Barbagini, F.; Calleja, E.; Strassburg, M. Phys. Status Solidi RRL 2013, $7,800-814$

(22) Tourbot, G.; Bougerol, C.; Grenier, A.; Den Hertog, M.; SamGiao, D.; Cooper, D.; Gilet, P.; Gayral, B.; Daudin, B. Nanotechnology 2012, 22, 1-8.

(23) Wernicke, T.; Schade, L.; Netzel, C.; Rass, J.; Hoffmann, V.; Ploch, S.; Knauer, A.; Weyers, M.; Schwarz, U.; Kneissl, M. Semicond. Sci. Technol. 2012, 27, 1-7.

(24) Pereira, S.; Correia, M. R.; Pereira, E.; O’Donnell, K. P.; TragerCowan, C.; Sweeney, F.; Alves, E. Phys. Rev. B 2001, 64, 205311.

(25) Kong, X.; Albert, S.; Bengoechea-Encabo, A.; Sánchez-García, M. A.; Calleja, E.; Trampert, A. Phys. Status Solidi A 2015, 212, 736739.

(26) Koblmüller, G.; Gallinat, C. S.; Speck, J. S. J. Appl. Phys. 2007, $101,083516$.

(27) Gallinat, C. S.; Koblmüller, G.; Brown, J. S.; Speck, J. S. J. Appl. Phys. 2007, 102, 064907.

(28) Averbeck, R.; Riechert, H. Phys. Status Solidi A 1999, 176, 301305 .

(29) Foxon, C. T.; Novikov, S. V.; Hall, J. L.; Campion, R. P.; Cherns, D.; Griffiths, I.; Khongphetsak, S. J. Cryst. Growth 2009, 311, 3423-3427.

(30) Lymperakis, L.; Neugebauer, J. Phys. Rev. B 2009, 79, 241308.

(31) Sekiguchi, H.; Kishino, K.; Kikuchi, A. Appl. Phys. Lett. 2010, 96, 231104.

(32) Bengoechea-Encabo, A.; Albert, S.; Lopez-Romero, D.; Lefebvre, P.; Barbagini, F.; Torres-Pardo, A.; Gonzalez-Calbet, J. M.; Sánchez-Garcia, M. A.; Calleja, E. Nanotechnology 2014, 25, 1-7. 